\title{
BMJ Open Comparison of propofol and dexmedetomidine infused overnight to treat hyperactive and mixed ICU delirium: a protocol for the Basel ProDex clinical trial
}

To cite: Hollinger A, Ledergerber $\mathrm{K}$, von Felten $\mathrm{S}$, et al. Comparison of propofol and dexmedetomidine infused overnight to treat hyperactive and mixed ICU delirium: a protocol for the Basel ProDex clinical trial. BMJ Open 2017;7:e015783. doi:10.1136/ bmjopen-2016-015783

- Additional material is published online only. To view please visit the journal online (http://dx.doi.org/10.1136/ bmjopen-2016-015783).

Received 2 January 2017 Revised 17 March 2017 Accepted 20 March 2017

\section{CrossMark}

${ }^{1}$ Department of Anaesthesia, Surgical Intensive Care, Prehospital Emergency Medicine and Pain Therapy, University Hospital Basel, Basel, Switzerland

${ }^{2}$ Department for Clinical Neurophysiology, Epilepsy and Movement Disorders, University Hospital Basel, Basel, Switzerland

${ }^{3}$ Department of Clinical Research, University Hospital Basel, Basel, Switzerland

${ }^{4}$ Medical Intensive Care Unit, University Hospital Basel, Basel, Switzerland

Correspondence to Dr Alexa Hollinger; alexa. hollinger@usb.ch

\section{ABSTRACT}

Background/objectives Delirium is a neurobehavioural disturbance that frequently develops particularly in the intensive care unit (ICU) population. It was first described more than half a century ago, where it was already discovered as a state that might come along with serious complications such as prolonged ICU and hospital stay, reduced quality of life and increased mortality. However, in most cases, there is still lack of proof for causal relationship. Its presence frequently remains unrecognised due to suggested predominance of the hypoactive form. Furthermore, in the general ICU population, it has been shown that the duration of delirium is associated with worse long-term cognitive function. Due to the multifactorial origin of delirium, we have several but no incontestable treatment options. Nonetheless, delirium bears a high burden for patient, family members and the medical care team. The Basel ProDex Study targets improvement of hyperactive and mixed delirium therapy in critically ill patients. We will focus on reducing the duration and severity of delirium by implementing dexmedetomidine into the treatment plan. Dexmedetomidine compared with other sedatives shows fewer side effects representing a better risk profile for delirium treatment in general. This could further contribute to higher patient safety. The aim of the BaProDex Trial is to assess the superiority of dexmedetomidine to propofol for treatment of hyperactive and mixed delirium in the ICU. We hypothesise that dexmedetomidine, compared with propofol administered at night, shortens both the duration and severity of delirium.

Methods/design The Basel ProDex Study is an investigator-initiated, one-institutional, two-centre randomised controlled clinical trial for the treatment of delirium with dexmedetomidine versus propofol in 316 critically ill patients suffering from hyperactive and mixed delirium. The primary outcome measure is delirium duration in hours. Secondary outcomes include deliriumfree days at day 28 , death at day 28 , delirium severity, amount of ventilator days, amount of rescue sedation with haloperidol, length of ICU and hospital stay, and pharmaceutical economic analysis of the treatments.

\section{Strengths and limitations of the study}

- The study's main strength is the implementation of a promising and more secure method of therapy for an unsolved problem: the lack of adequate and secure therapy of delirium, a condition first described more than five decades ago. Dexmedetomidine compared with other sedatives shows fewer side effects, mainly bradycardia and hypotension. Evidence strongly suggests a high benefit for treatment of delirium going along with increased comfort and safety of critically ill patients. In addition, no severe adverse events are expected by its use for sedation in delirious patients after careful enrolment following our exclusion criteria.

- Night-time infusion may normalise the day/night cycle of the patients, mimicking a more physiological circadian rhythm.

- The Basel ProDex Study will primarily recruit from two intensive care units (two in Switzerland) to achieve the calculated sample size within the foreseen time period.

- The study is limited by the heterogeneous general condition and past medical history of critically ill patients. These variable conditions will be addressed due to assessment and analysis of the Simplified Acute Physiology Score II score.

Sample size was estimated to be able to show the superiority of dexmedetomidine compared with propofol regarding the duration of delirium in hours. The trial will be externally monitored according to good clinical practice (GCP) requirements. There are no interim analyses planned for this trial.

Ethics and dissemination This study will be conducted in compliance with the protocol, the current version of the Declaration of Helsinki, the International Conference on Harmonization- Good Clinical Practice (ICH-GCP) or Europäische Norm International Organization for Standardization (ISO EN 14155; as far as applicable) as well as all national legal and regulatory requirements. 
Only the study team will have access to trial specific data. Anonymisation will be achieved by a unique patient identification code. Trial data will be archived for a minimum of 10 years after study termination. We plan to publish the data in a major peer-reviewed clinical journal.

Trials registration ClinicalTrials.gov Identifier: NCT02807467

Protocol version Clinical Study Protocol Version 2, 16.08.2016

\section{BACKGROUND AND RATIONALE}

Dexmedetomidine is a potent selective $\alpha$ - 2 adrenergic receptor agonist frequently used for sedation in the intensive care unit (ICU). It promotes sedation, anxiolysis and moderate analgesia with minimal respiratory depression. ${ }^{1}$ Sedation of ICU patients remains challenging for doctors and nurses as there is evidence that the administration of sedatives in critically ill patients is potentially harmful, mainly in relation to delirium during critical care and the subsequent clinical outcome. ${ }^{2}$ However, in many cases, sedation is supportive for both patients and ICU personnel allowing controlled interactions with the patient through established comfort and security. ${ }^{3}$ This supports the patient's autonomy and establishes a less threatening environment. Delirium is frequent in patients in the ICU and in hospitalised patients who are acutely ill, especially after cardiac or orthopaedic surgery and is associated with adverse outcome. ${ }^{4}$ It is a pathological neurobehavioural syndrome caused by transient alteration of the normal neuronal network activity secondary to systemic disturbances. ${ }^{5}$

In the ICUs of the University Hospital of Basel, standard therapy of hyperactive delirium phases consists of the intravenous or oral administration of haloperidol and oral quetiapine. At present, despite the recommendation against its use in the latest American guidelines for sedation, analgesia and delirium ${ }^{6}$ and ongoing concern for its safety and efficacy, ${ }^{7}$ haloperidol is the first-line agent used worldwide for the treatment of delirium in general. ${ }^{89}$ Nevertheless, there seems to be evidence for its potential to prevent delirium. ${ }^{10}$

Due to disturbed circadian rhythm among patients suffering from delirium, exclusive sedation with haloperidol and quetiapine, especially at night, is insufficient and leads to daytime sedation and sleepiness, and additional sedative agents such as propofol are frequently required (table 1). However, there has been evidence for a better and safer alternative: dexmedetomidine. This has been proven in the cohort of patients who underwent cardiac surgery $^{11}$ where perioperative use of dexmedetomidine was associated with decreased mortality up to 1 year and decreased incidence of postoperative complications and delirium. A study investigating the effect of dexmedetomidine in addition to standard care alone (placebo) on ventilator-free time in patients with agitated delirium revealed findings of more ventilator-free hours at 7 days supporting the use of dexmedetomidine for sedation in patients suffering from delirium who depend on the ventilator. ${ }^{12}$ Teegarden and Prough suggested that dexmedetomidine could be the next step after failure of haloperidol for delirium treatment. ${ }^{13}$ They referred to the non-randomised controlled trial performed by Carrasco and colleagues, who investigated dexmedetomidine for the treatment of hyperactive delirium refractory to haloperidol in non-intubated patients in the ICU. They declared dexmedetomidine as a drug that possesses all favourable properties to serve as an ideal treatment for ICU-associated delirium and agitation: relieve of symptoms without causing excessive sedation, fewer side effects than haloperidol, little interaction with other drugs, and easy to titrate. ${ }^{14}$ Other publications ${ }^{1516}$ suggested dexmedetomidine to be a valuable sedative agent in the ICU population in reducing ICU length of stay and time to extubation but did not specify on delirium.

Because dexmedetomidine induces a unique sleep-like sedation state, ${ }^{17}$ it could have beneficial effects even on the disturbed circadian rhythm when infused at night. In highly selected patients, night-time dexmedetomidine infusions to induce light sedation could be shown to increase sleep efficiency and shift the 24-hour sleep pattern mainly to the night. ${ }^{18}$ The mechanism of action of dexmedetomidine is unique compared with traditionally administered sedative agents due to its lack of activity at the $\gamma$-aminobutyric acid receptor and missing anticholinergic activity. ${ }^{1}$ Possible pathophysiological explanations for the development of delirium have been considered to stem from neurotransmitter disturbances termed 'neurotransmitter hypothesis' and include dysfunction of cholinergic transmission. ${ }^{19}$ So both suggested that beneficial impact on disturbed circadian rhythm and lack of anticholinergic activity are promising features of dexmedetomidine for delirium treatment. The former explains our proposition to infuse dexmedetomidine only during nightly hours in the study group.

\section{HYPOTHESIS}

In our prospective, randomised controlled study, we aim to test the hypothesis that continuous infusions of dexmedetomidine compared with propofol between 20:00 and 06:00 after diagnosis of hyperactive or mixed delirium may help reinstitute a normal day-night cycle mimicking a more physiological circadian rhythm due to the sleeplike sedative effect of dexmedetomidine, and therefore decrease the duration of delirium.

\section{METHODS}

\section{Trial design}

The Basel ProDex Study is an investigator-initiated, one-institutional, two-centre, prospective randomised controlled clinical trial of patients suffering from hyperactive or mixed delirium.

\section{Approvals}

Approval to conduct this study was granted by the Ethics Committee of Northwestern and Central Switzerland (EKNZ 2016-00843) in September 2016. The study is registered at the Swiss National Clinical Trial Portal 


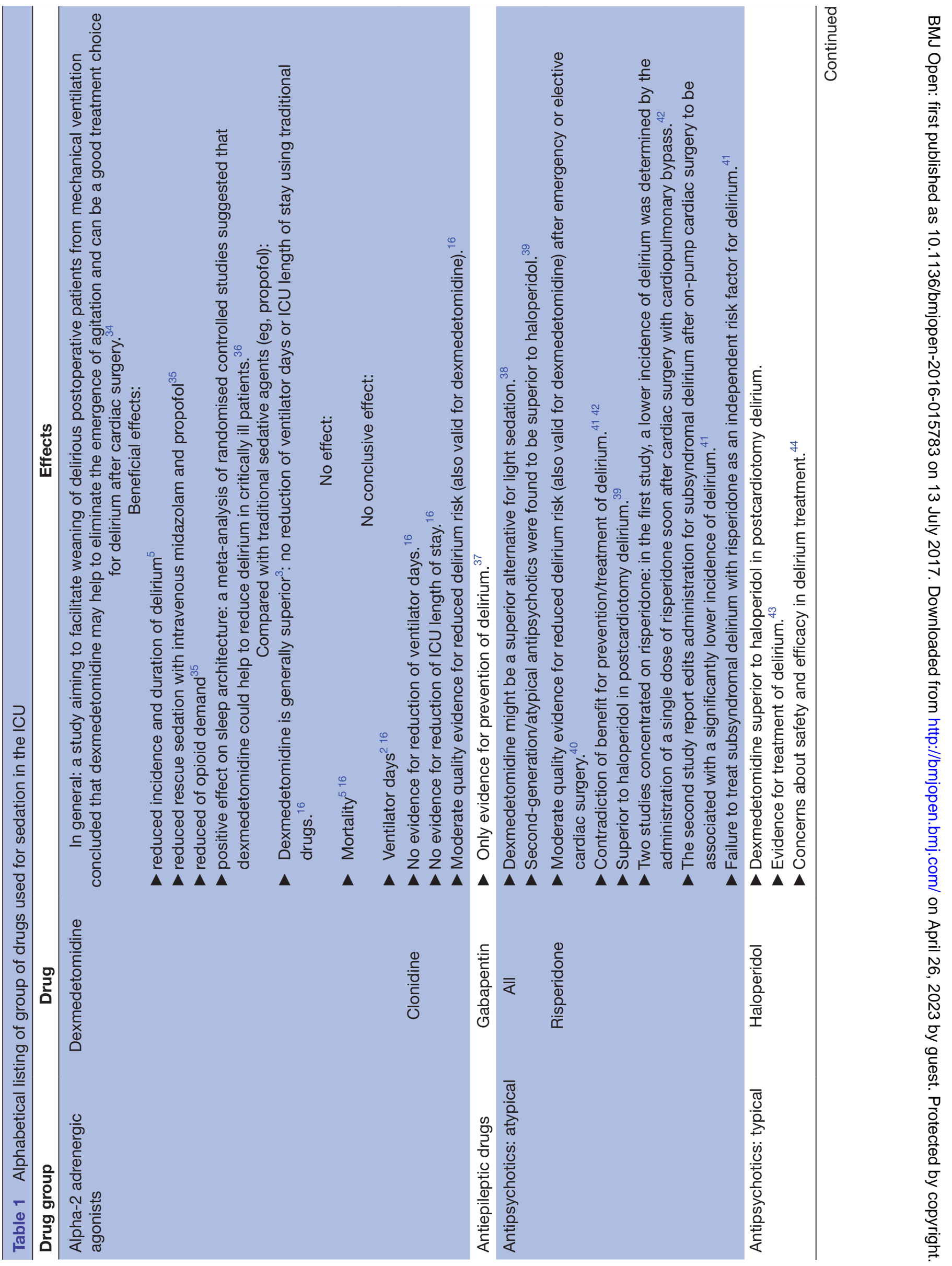




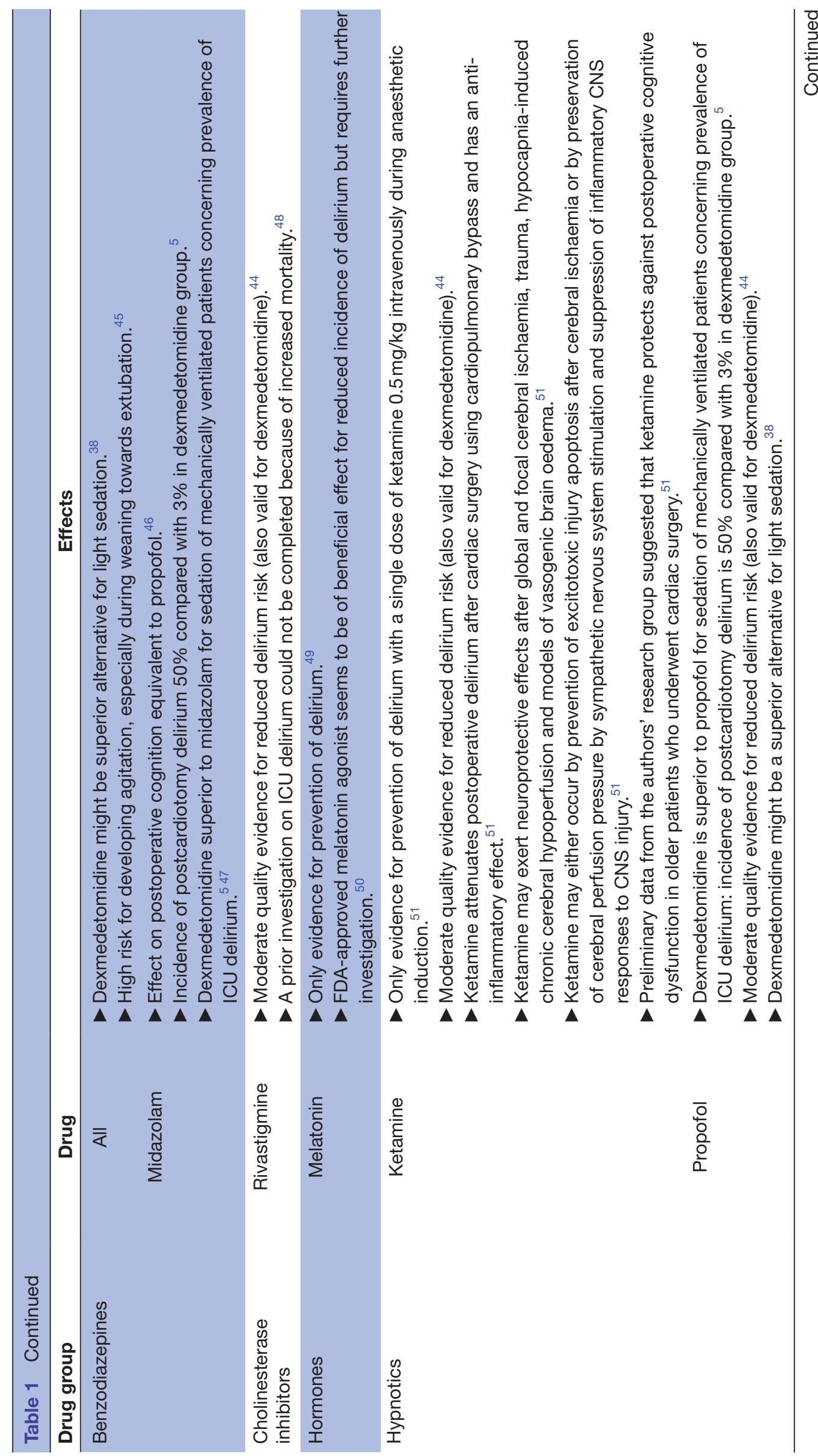




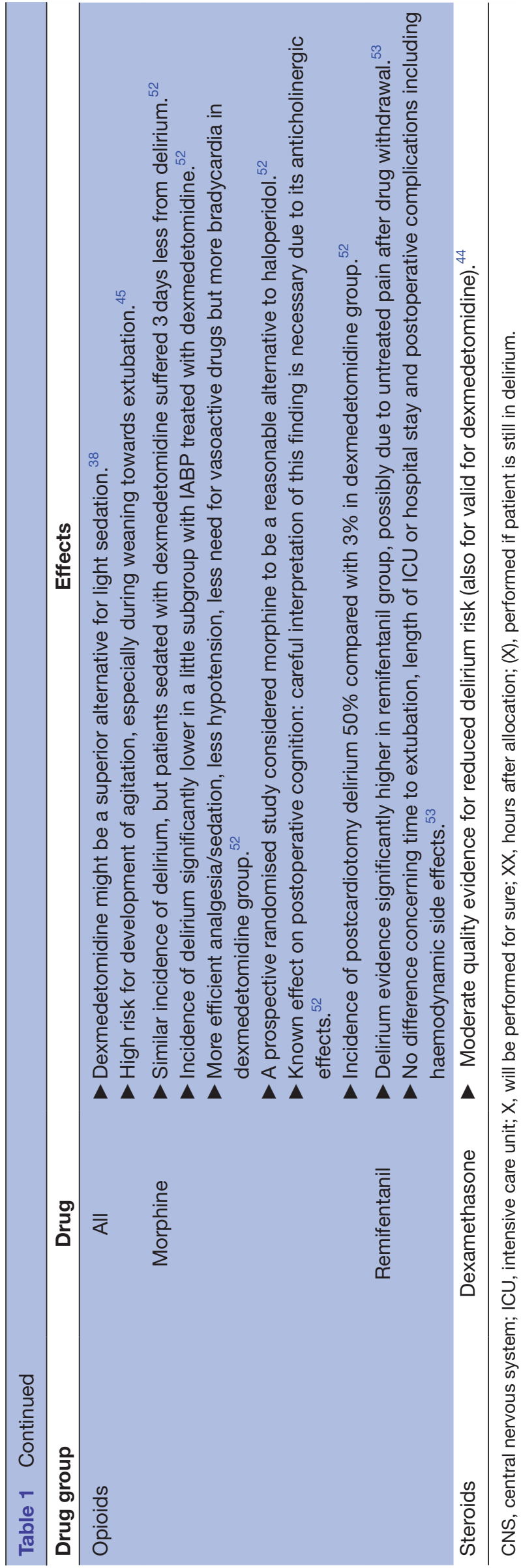

(identifier: SNCTP000001922) and at ClinicalTrials.gov (identifier: NCT02807467).

Study setting

Adult ICU admitting medical or surgical patients.

Study population

Inclusion criteria

Participants fulfilling the following inclusion criteria are eligible for the study:

- Adult patients (age 18 years or older)

- Current delirium (hyperactive or mixed type) detected by a specialised assessment method (Intensive Care Delirium Screening Checklist (ICDSC) in one of the participating ICUs.

\section{Exclusion criteria}

Participants meeting the following criteria are excluded from the study:

- Delirium prior to ICU admission

- $\mathrm{Egg}^{20}$ and soy allergy

- Hypersensitivity to the active substances

- Advanced heart block (grade 2 or 3) unless paced

- Bradycardia of different origin

- Uncontrolled hypotension

- Acute cerebrovascular conditions

- Severe cardiac dysfunction

- Age $<18$ years

- Terminal state

- Pregnancy

- Status epilepticus or postictal states following seizures on electroencephalogram (EEG)

- Active psychosis

- Delirium tremens

- Substance abuse with experience of acute withdrawal.

\section{TRIAL MEDICATION}

Prior to the first nightly infusion of dexmedetomidine or propofol that represents the period in which hyperactive or mixed delirium is diagnosed and delirium treatment is initiated, the administration of sedatives follows the defined ICU treatment algorithm of the ICUs of the University Hospital Basel: in the acute setting, intravenous haloperidol is administered. This is followed by oral quetiapine or, in case of pre-existing disturbed cerebral performance, trazodone hydrochloride.

Patients enrolled in the trial will be randomised to receive either dexmedetomidine (Dexdor, concentrated $200 \mu \mathrm{g} / 2 \mathrm{~mL}$ for intravenous administration, Orion Pharma AG, Orion Corporation, Espoo, Finland) or propofol (Propofol Lipuro 1\% 1 g/100 mL for intravenous administration, B. Braun Medical AG, Sempach, Switzerland) administered by continuous infusion from 20:00 to 06:00 beginning the evening after diagnosis of hyperactive or mixed delirium. After dexmedetomidine or propofol infusion over the previously described period of time, we shall exclusively use haloperidol intravenously (Haldol, Janssen-Cilag AG, Schaffhausen, Switzerland) 
as rescue medication and nursing care for further treatment of delirium during daytime. During the consecutive time frame between 20:00 and 06:00, the randomised study drug will be infused again if indicated. The latter will allow us to clearly detect the suggested shortening of delirium duration in the cohort where dexmedetomidine is being used as sedative agent. If intravenous sedation is needed during daytime because of aggressive behaviour, the patient will receive the assigned drug. The amount of daytime sedation will be recorded.

After randomisation, the doctor responsible for the patient will prescribe the study drug, and the nurse caring for the patient will then prepare and administer the study drug as prescribed:

- Dexmedetomine: $0.7 \mu \mathrm{g} / \mathrm{kg}$ bolus, followed by $0.2-$ $1.4 \mu \mathrm{g} / \mathrm{kg} / \mathrm{hour}$ for desired level of sedation

- Propofol: 1-4mg/kg/hour for desired level of sedation.

The indicated dosage of dexmedetomidine and propofol usually is sufficient for sedation in patients who are agitated. However, if this is not the case that haloperidol can also be used as rescue medication at night.

There will be no continuous infusion of propofol/ dexmedetomidine during the day. If by solely administration of haloperidol the hyperactive patient cannot be controlled ,additional sedatives will be allowed (ie, oral quetiapine and propofol by bolus).

\section{OUTCOME MEASURES}

\section{Primary outcome measure}

Delirium duration in hours.

\section{Secondary outcome measures}

- Delirium free days at 28 days

- Death until day 28

- Severity of ICU delirium (sum of highest ICDSC scores per nursing shift divided by the number of shifts)

- Number of ventilator days

- Need for rescue sedation (amount of haloperidol in milligram)

- Amount of oral quetiapine

- Total costs of medication (dexmedetomidine infused + rescue medication or propofol infusion + rescue medication)

- Length of ICU stay (hours)

- Length of hospital stay (days)

- Depth of sedation in both groups (median Richmond Agitation Sedation Scale (RASS) score or median Sedation Agitation Scale (SAS) score; proven similar rates of delirium assessment when confusion assessment method-ICU (CAM-ICU) is used ${ }^{21}$

- Depth of sedation in study group (determined by EEG analysis).

\section{DEFINITIONS/CONDITIONS}

Inclusion criteria

The criteria for the diagnosis of delirium are ${ }^{22}$ :
- acute beginning and fluctuating course

- absence of known psychiatric disorder

- disturbance of awareness and attention

- ongoing disturbance of perception of fluctuating course

- psychomotoric disturbances (hyperactive or quiet)

- mostly no isolated connection to an acute mental strain or pre-existing psychiatric disease.

Three subtypes of delirium, hypoactive, hyperactive and mixed, were classified more than 30 years ago based on the clinical presentation focused on psychomotor behaviour. $^{23}$

Hyperactive delirium presents with restlessness, agitation and hypervigilance and is often accompanied by hallucinations and delusions. Patients showing lethargy and who seem slowed down (eg, speech and spontaneous movements) raise suspicion for development of hypoactive delirium. Mixed delirium shows features of both conditions. $^{24}$

\section{Exclusion criteria}

Hypersensitivity to the active substances is defined as known allergy to one of the study drugs.

Egg and soy allergy: Even though patients who are allergic to eggs are generally allergic to egg protein or albumin, not lecithin representing the egg phosphatides which are present in the propofol emulsion, an adverse allergic reaction to propofol in a patient with egg hypersensitivity has been reported. ${ }^{20}$ There is no clear evidence to determine propofol administration as a contraindication in a patient with history of egg allergy, ${ }^{25}$ but we will exclude these patients for safety reasons.

Cardiac rhythm: The patient has to be excluded if an advanced heart block (grade 2 or 3 ) is seen in the ECG or if a bradycardia of different origin is documented or assessed, unless the patient has a pacemaker.

Uncontrolled hypotension is defined as systolic pressure $<30 \%$ from baseline or mean arterial pressure $<60 \mathrm{~mm}$ $\mathrm{Hg}$ that cannot be controlled by noradrenaline $<0.1 \mu \mathrm{g} /$ $\mathrm{kg} / \mathrm{min}$. Uncontrolled hypotension, as defined in the protocol, leads to exclusion of the study patient. In case of hypotensive blood pressure values above our definition of uncontrolled hypotension, we would reduce the drug dose, and this would not lead to study exclusion.

Acute cerebrovascular conditions include acute vascular ischaemic events or acute intracranial haemorrhage of traumatic origin. Hence, we will include patient's suffering from chronic subdural haematoma.

Severe cardiac dysfunction is characterised by decreased contractility and impaired fluid responsiveness or dilated ventricles (eg, $\mathrm{EF}<30 \%$ ). This will be assessed according to clinical development and echocardiography.

Age < 18 years: We will only include adult patients in our study.

Terminal state: Patients suffering from an incurable disease and who have a terminal illness will not be included in our study. 
Pregnancy: A negative pregnancy test will be necessary for study inclusion in women aged $\leq 45$ years.

Status epilepticus or postictal states following seizures on EEG: An unexplained unconscious state will be evaluated with EEG prior to study enrolment.

Active psychosis is of non-organic origin (classified as functional disturbance). Active psychosis will be diagnosed/excluded according to the following criteria:

- crescendo development over days to weeks (contrary to delirium which usually develops acutely within minutes)

- nodetectableorganic causefor delusion, hallucinations and other types of perceptual disturbances, or for severe behavioural disturbance

- no disturbed vigilance at the beginning

- perceptual disturbances mostly discrete or of short duration (not sufficient for diagnosis of delirium)

- often connected to acute mental strain or pre-existing psychiatric disease.

Delirium tremens: Delirious symptoms that raise suspicion for a delirium due to substance withdrawal, such as visual or tactile hallucinations, body tremors, sensitivity to stimuli (light/sounds/touch).

Substance abuse with experience of acute withdrawal: Depending on the substance (ie, benzodiazepines and antipsychotics) patients might be excluded during the screening procedure. If the study participant raises suspicion for substance withdrawal (eg, completion of patient history by next of kin), he or she will be withdrawn from the study.

Desired level of sedation

SAS: 3/RASS: 0.

\section{Primary outcome measure}

Duration of delirium in hours: the onset of delirium will be defined as the start of the first of a minimum of two subsequent shifts with an ICDSC $\geq 4$ and an SAS $>4$ or
RASS $>1$. The end of the delirium will be defined as the end of the last shift with an ICDSC $\geq 4$ that precedes a minimum of two subsequent shifts with an ICDSC $<4$.

The RASS and the SAS can be extrapolated to one another as follows:

\begin{tabular}{lcc}
\hline Action/interpretation & RASS & SAS \\
\hline Observation & $\leq-3$ & $<3$ \\
Screening for delirium & $>-3$ & $\geq 3$ \\
Severe agitation & $2-4$ & $6-7$ \\
\hline
\end{tabular}

Whereas the RASS score includes 10 levels compared with 7 levels in the SAS, the RASS is more precise in the determination of the level of agitation. However, both scores are used in the ICU. Due to lack of data, no superiority of one score over the other was shown until now. As mentioned above, with the CAM-ICU, no relevant difference for delirium assessment has been found. Because of a long-time experience in our institution and its high sensitivity and specificity as described later, the ICDSC represents the preferred assessment tool in our study.

\section{Serious adverse event}

Serious adverse events (SAEs) are classified as any medical occurrence that results in death, is life threatening, requires prolongation of existing hospitalisation or results in persistent or significant disability/incapacity.

\section{Serious unexpected adverse drug reaction}

A serious unexpected adverse drug reaction (SUSAR) indicates an adverse drug reaction that is of a nature or severity that is not consistent with the applicable product information

\section{STUDY PERIOD OVERVIEW}

The study period consists of enrolment, allocation, postallocation and closeout (table 2). Allocation is defined as

Table 2 Study period overview

Study period

\begin{tabular}{|c|c|c|c|c|c|c|}
\hline & Enrolment & Allocation & Postallocation & & & Closeout \\
\hline Time point (hours) & $-\mathrm{t} 1$ & $t_{0}=0$ & $t 1=24$ & $\mathrm{~T} 2=48$ & $\mathrm{~T} 3=72$ & T4=XX \\
\hline Enrolment & $x$ & & & & & \\
\hline Informed consent & $x$ & & & & & \\
\hline
\end{tabular}

\section{Assessments}

\begin{tabular}{|c|c|c|c|c|c|c|}
\hline Vital signs & & $x$ & $x$ & $X$ & $x$ & $x$ \\
\hline Delirium screening tools & $x$ & $x$ & $x$ & $x$ & $x$ & $x$ \\
\hline \multicolumn{7}{|l|}{ Drug therapy } \\
\hline Rescue medication & & $\mathrm{X}$ & $(X)$ & $(X)$ & $(X)$ & \\
\hline
\end{tabular}

$X=$ will be performed for sure; $X X=$ hours after allocation; $(X)=$ performed if patient is still in delirium. 


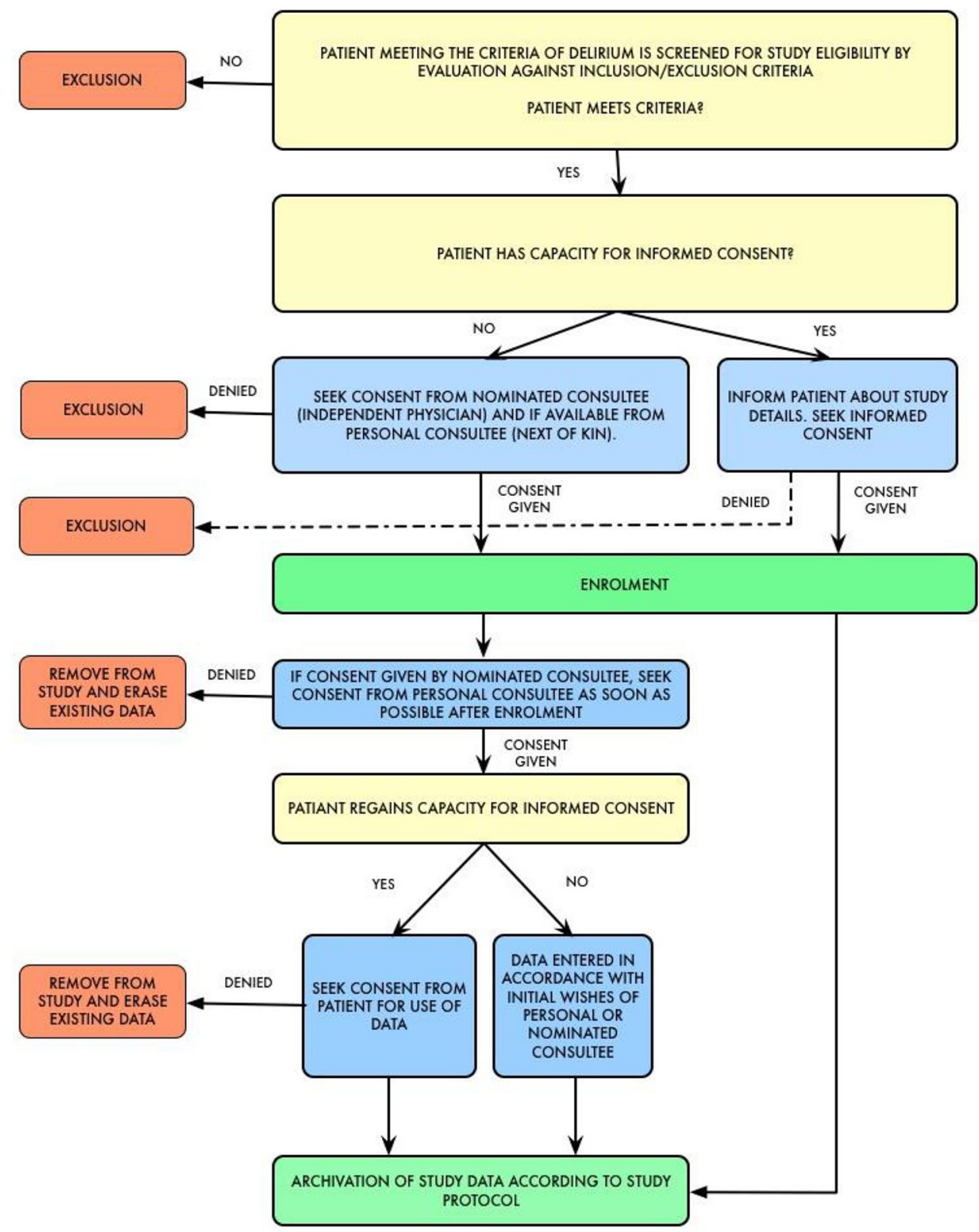

Figure 1 Allocation overview.

the first day of dexmedetomidine or propofol infusion from 20:00 to 06:00 after diagnosed delirium. Closeout is defined as point of time of delirium recovery.

\section{Screening}

Following the delirium assessment part of the assess and treat pain, Awakening and Breathing trials, Coordination of care and Choice of sedative, Delirium Monitoring and Management and Early Mobility bundle ${ }^{26}$ representing the core of the institutional pain, agitation and delirium (PAD) guidelines, ${ }^{27}$ we will screen every patient admitted to the ICU for ongoing delirium to evaluate eligibility for study recruitment (figure 1).

\section{Delirium assessments}

Every patient admitted to the trial site will be screened for study eligibility following the inclusion and exclusion criteria. Patients meeting study participation criteria are those who fulfil the inclusion criteria and none of the exclusion criteria. Eligibility screening data will be stored using the electronic case report form established by the Clinical Trial Unit (CTU; part of Department of Clinical Research), Basel. In parallel, this will allow the opportunity for an intention-totreat (ITT) analysis.

For screening and for the whole duration of the delirium, we will assess the ICDSC during every shift. The ICDSC and the CAM-ICU are the most well-studied and widely implemented adult ICU delirium screening tools worldwide and the two delirium screening tools recommended by recently updated clinical practice guidelines. The ICUs of the University Hospital Basel routinely use the ICDSC for assessment of delirium. 
Both clinical scoring systems have been recommended for the screening of delirium in ICU by the Society of Critical Care Medicine based on high-quality evidence. ${ }^{6}$ Direct comparisons of the diagnostic accuracy of the CAM-ICU and the ICDSC have been performed in recent studies with heterogeneous ICU populations revealing a higher sensitivity and specificity of the ICDSC than the CAM-ICU. ${ }^{28-30}$

According to the studies and guidelines mentioned above, an ICDSC $\geq 4$ was defined as delirium. ${ }^{631}$

The ICDSC is an eight-item checklist of delirium symptoms evaluated over an 8-24 hour period. Patients are given one point for each symptom that manifests during the specified time frame (zero points if a symptom did not manifest). The eight symptoms are: level of consciousness, inattention, disorientation, hallucinations /delusions/psychosis, psychomotor agitation or retardation, inappropriate speech or mood, sleep/wake cycle disturbances and symptom fluctuation. A score $\geq 4$ indicates a positive ICDSC and the presence of delirium. Key symptoms of delirium can be part of a focused evaluation by the bedside clinician. For example, as the nurse introduces himself/herself to the patient and performs the clinical assessment he/ she also looks for signs that may indicate the patient is inattentive, has disorganised thinking, psychomotor agitation/retardation, and so on. Presence of any symptoms noted during an initial focused evaluation can immediately be scored on the ICDSC. The patient can subsequently be observed and scored for additional symptoms that manifest or fluctuate during the remainder of the specified time period. Without objective criteria, there could be variation in how symptoms are identified in intubated patients. See table 3 for suggestions on how to assess delirium symptoms in this special population using the ICDSC. ${ }^{32}$

\section{Three and 12 months' follow-up}

To also assess long-term follow-up of patients that received dexmedetomidine and compare it with those who received propofol, we will perform a follow-up 3 and 12 months after the prevailing hospital case has been officially closed (discharge date). By this follow-up we will assess the following information equally at 3 and 12 months:

- death during hospital stay

- death after hospital discharge

- hospital readmission

- activities of daily life questionnaire.

\section{ASSESSMENTS}

\section{Assessment of delirium}

Delirium will be assessed by the ICDSC as explained above.

\section{Assessment of sedation and pain level}

For assessment of sedation level, we will comply with the SAS and the RASS, and for pain with the Critical Care
Pain Observational Tool and/or Visual Analogue Scale/ Numeric Rating Scale (VAS/NRS).

All scores (ie, ICDSC, RASS/SAS, CPOT and VAS/NRS) are assessed by the treating nurses of the study patient. In the centres involved, advanced nurse practitioners coach the nursing staff and checks agitation and delirium assessments in the study patients in regular intervals.

\section{Assessment of study drug side effects}

To evaluate potential side effects, we will record the following parameters:

- heart rate

- blood pressure

- fluid balance

- blood count

- creatinine

- blood urea nitrogen

- triglycerides

Blood pressure (lowest systolic pressure and lowest mean arterial pressure) and heart rate will be carefully monitored to detect the most common side effect of dexmedetomidine and propofol: hypotension and bradycardia. A heart rate below $40 / \mathrm{min}$ or a systolic pressure of $<20 \%$ from baseline will indicate the need to evaluate safety of continued study drug administration.

Creatine kinase, myoglobin and lactate will be also monitored to detect propofol-related infusion syndrome (PRIS), a threatening side effect of long-term propofol infusion. If the latter-mentioned values are elevated, we will discontinue the drug. If after discontinuation of the drug we measure further elevation of these values, we will perform a muscle biopsy, if the syndrome leads to haemodynamic abnormalities, lactic acidosis and rhabdomyolysis.

\section{Electroencephalography}

EEG evaluation will enable us to analyse different patterns of sleep architecture under the influence of dexmedetomidine.

Former investigations were able to show, after analysis of density, duration, amplitude and frequency of sleep spindles, that EEG activity under dexmedetomidine sedation is quite similar to the normal sleep pattern of the physiological sleep state N2 with light to moderate appearance of slow-wave activity and a lot of sleep spindle activity. Within quantitative EEG analyses, the sleep spindles were alike during sedation with dexmedetomidine and normal sleep. This finding supports prior evidence of activation of normal non-rapid eye movement sleep-promoting pathways caused by the sedative agent that will be further investigated in our trial. ${ }^{17}$

\section{Assessments documented on the case report form}

On our case report form, we will document the following information on our study participants (sequential order):

- patient information

- study group

- study eligibility 
Table 3 Suggestions for assessing delirium with the ICDSC ${ }^{54}$

1. Altered level of consciousness: choose one from $A$ to $E$

\begin{tabular}{|c|c|c|}
\hline A. Exaggerated response to normal stimulation & $\mathrm{SAS}=5,6,7$ or $\mathrm{RASS}=+1$ to +4 & (1 point) \\
\hline B. Normal wakefulness & $\mathrm{SAS}=4$ or $\mathrm{RASS}=0$ & (0 points) \\
\hline C. Response to mild or moderate stimulation (follows commands) & $\mathrm{SAS}=3$ or $\mathrm{RASS}=-1$ to -3 & (0 points) \\
\hline $\begin{array}{l}\text { D. Response only to intense and repeated stimulation (eg, loud } \\
\text { voice and pain) }\end{array}$ & $\mathrm{SAS}=2$ or $\mathrm{RASS}=-4$ & Stop assessment \\
\hline E. No response & $\mathrm{SAS}=1$ or $\mathrm{RASS}=-5$ & Stop assessment \\
\hline 2. Inattention & & (1 point if any present) \\
\hline \multicolumn{3}{|l|}{ A. Difficulty in following commands or } \\
\hline \multicolumn{3}{|l|}{ B. Easily distracted by external stimuli or } \\
\hline C. Difficulty in shifting focus & & \\
\hline
\end{tabular}

Does the patient follow you with their eyes?

$\begin{array}{ll}\text { 3. Disorientation } & \text { (1 point if any } \\ \text { abnormality) }\end{array}$

A. Mistake in either time, place or person

Does the patient recognise ICU caregivers who have cared for him/her and not recognise those that have not? What kind of place are you in? (list examples)

\section{Hallucinations or delusions}

(1 point if any abnormality)

A. Equivocal evidence of hallucinations or a behaviour due to hallucinations (hallucination=perception of something that is not there with NO stimulus) or

B. Delusions or gross impairment of reality testing (delusion=false belief that is fixed/unchanging)

Any hallucinations now or over past 24 hours? Are you afraid of the people or things around you? (fear that is inappropriate to the clinical situation)
5. Psychomotor agitation or delay
(1 point for either)

A. Hyperactivity requiring the use of additional sedative drugs or restraints in order to control potential danger (eg, pulling out intravenous lines or hitting staff) or

B. Hypoactive or clinically noticeable psychomotor slowing or delay

Based on documentation and observation during shift by primary caregiver

6. Inappropriate speech or mood $\quad$ (1 point for either)

A. Inappropriate, disorganised or incoherent speech or

B. Inappropriate mood related to events or situation

Is the patient apathetic to current clinical situation (ie, lack of emotion)? Any gross abnormalities in speech or mood? Is patient inappropriately demanding?

\section{Sleep/wake cycle disturbance}

(1 point for any

abnormality)

A. Sleeping less than four hours at night or

B. Waking frequently at night (does not include wakefulness initiated by medical staff or loud environment) or

C. Sleep $\geq 4$ hours during day

Based on primary caregiver assessment

\section{Symptom fluctuation}

(1 point for any)

Fluctuation of any of the above items (ie, 1-7) over 24 hours (eg, from one hospital shift to another)

Based on primary caregiver assessment

Total ICSDC score (add 1-8)

ICDSC, Intensive Care Delirium Screening Checklist; ICU, intensive care unit; RASS, Richmond Agitation Sedation Scale; SAS, Sedation

Agitation Scale. 


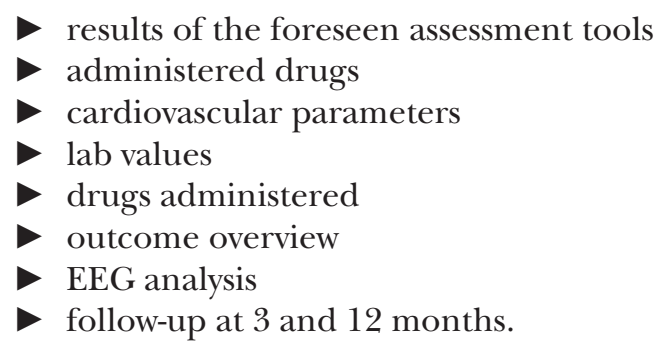

\section{RANDOMISATION}

Trial staff will have access $24 / 7$ to the electronic case report form where patients are screened and randomised to one of the trial arms. Randomisation will be performed by the CTU Basel. A unique patient identification code will be assigned to every screened patient consisting of the patient's initials, year of birth, gender and type of delirium (ie, $\mathrm{H}=$ hyperactive and $\mathrm{M}=$ mixed). Since every screened patient will be assessed using our case report form, we may replace the ' $\mathrm{H}$ ' or ' $\mathrm{M}$ ' (type of delirium) with ' $W$ ' to indicate patient withdrawal after assessment of exclusion criteria.

Stratified block randomisation will be performed with stratification for gender, treating unit, sepsis and heart surgery.

We will randomise every hyperactively delirious patient to our study. For the mixed delirium, it follows that such a patient would be recruited at the first point of time he or she shows agitation assessed by the RASS/SAS score. Study patients do not have to be hyperactive for a minimum number of assessments.

\section{BLINDING}

Not applicable. Potential drug side effects are drug specific.

The treating medical team as well as the study team will not be blinded for the outcome assessment. The statistician who will analyse the final data will be blinded for study drug medication as far as applicable (eg, not possible for EEG analysis).

\section{PATIENT INFORMATION AND INFORMED CONSENT}

Because all study participants are delirious, they do not have the capacity to give their consent for the study. For this reason, an independent auditing physician, acting as the patient's representative, will declare each patient's suitability for trial participation in the patient's name. The signed document of the independent physician is the prevailing condition for inclusion of the patient in our study.

If possible, depending on the general condition of the delirious participants, the investigators will explain to each participant the nature of the study, its purpose, the procedures involved, the expected duration, the potential risks and benefits and any discomfort it may entail. Each participant (depending on constitution) or the next of kin will be informed that the participation in the study is voluntary and withdrawal from the study is possible any time and that withdrawal of consent will not affect the subsequent medical assistance and treatment.

If anyhow possible, the participant or the next of kin must be informed that the medical record may be examined by authorised individuals other than their treating physician.

All participants in the study will be provided a participant information sheet describing the study and providing sufficient information for the participant and his/her next of kin to make an informed decision about their participation in the study. There will also be a consent form for the participant's next of kin.

The patient and next of kin information sheet and the consent forms have been submitted to the competent ethics committee for revision and have been approved. If possible, the formal consent of a participant's next of kin, using the approved consent form, must be obtained before the participant is subjected to any study procedure.

If possible, the next of kin should read and consider the statement before signing and dating the informed consent form and should be given a copy of the signed document. The consent form must also be signed and dated by the investigator (or his designee). The signed form will be retained as part of the study records.

After recovery, the patient will be informed about his/ her participation in the trial and he/she will have the possibility to withdraw their data from the study. In case of ex-post study withdrawal, patient data will be destroyed.

\section{SAFETY}

Since dexmedetomidine compared with other sedatives shows fewer side effects, mainly bradycardia and hypotension, and evidence strongly suggests high benefit for treatment of delirium going along with increased comfort and safety also for critically ill patients, no severe adverse events are expected by its use for sedation in delirious patients after careful enrolment following our exclusion criteria. We will monitor additional laboratory parameters for early detection of threats by long-term PRIS as mentioned above.

An individual subject will be excluded from the study if any of the following occur in the subject in question:

- withdrawal of consent by the independent physician or a next of kin

- an adverse event that in the opinion of the sponsor contraindicates further measuring (emergency setting).

\section{Categorisation of the study}

Dexmedetomidine comes under risk category A in our trial. It is a medicinal product authorised in Switzerland, and its use here is in accordance with the prescribing information: sedation in the ICU of patients aged $\geq 18$ years targeting arousability on verbal stimuli (RASS score 0 to -3$)$. 


\section{Serious adverse reactions}

The occurrence of SAEs will be assessed during every shift based on the bedside visit and study of vital and laboratory parameters and will be recorded daily on the electronic case report form.

All changes in research activity and unanticipated problems have to be reported to the competent Ethics Committee by the sponsor and the principal investigator. An SAE or SUSARs has to be reported within 7 days maximum if fatal, otherwise within 15 days. An annual safety report will be provided by the sponsor.

\section{Dexmedetomidine}

No SAEs are specified in the product characteristics of dexmedetomidine (https://pubchem.ncbi.nlm.nih.gov/ compound/dexmedetomidine) if used according to the indicated cautions:

- renal/hepatic impairment

- risk of hypotension, bradycardia and sinus arrest

- caution in cardiovascular disease, diabetes and in patients receiving vasodilators

- potential withdrawal symptoms if abruptly withdrawn after $>24$ hours of continuous use

- use beyond 24hours associated with tolerance, tachyphylaxis and dose-related increase in adverse effects (eg, ARDS, respiratory failure and agitation).

The only contraindication listed is hypersensitivity to the product. Adverse reactions are listed as follows:

- hypotension $(28 \%)$

- bradycardia (1\%-10\%)

- atrial fibrillation (1\%-10\%)

- anaemia (1\%-10\%)

- fever $(1 \%-10 \%)$

- pleural effusion (1\%-10\%)

- leucocytosis (1\%-10\%)

- pulmonary oedema $(1 \%-10 \%)$.

\section{Propofol}

Propofol is contraindicated under the following circumstances to avoid adverse reactions:

- lack of ventilatory support

- severe cardiac dysfunction

- documented hypersensitivity, egg allergy, soybean/soy allergy.

List of known adverse reactions:

- $>10 \%$ hypotension (adults 3\%-26\%)

- apnoea lasting 30-60s (adults 24\%)

- apnoea lasting $>60$ s (adults $12 \%$ )

- movement (adults 3\%-10\%)

- injection site burning/stinging/pain (adults 18\%)

- $1 \%-10 \%$ respiratory acidosis during weaning (3\%$10 \%)$

- hypertriglyceridaemia (3\%-10\%)

- rash (adults 1\%-3\%)

- pruritus $(1 \%-3 \%)$

- arrhythmia (1\%-3\%)

- bradycardia (1\%-3\%)
- cardiac output decreased (1\%-3\%; concurrent opioid use increases incidence)

- tachycardia (1\%-3\%)

- $<1 \%$ arterial hypotension, anaphylaxis, asystole, bronchospasm, cardiac arrest, seizures, opisthotic reaction, pancreatitis, pulmonary oedema, phlebitis, thrombosis and renal tubular toxicity.

\section{PATIENT WITHDRAWAL}

Patients withdrawn from the study will be included in the ITT analysis. Subjects who could not be followed over the intended period and all designated points of assessments, regardless of reason, will not be followed. Unless consent for follow-up is withdrawn, subjects discontinued before closeout will be followed for the full study period with all laboratory and clinical evaluations collected as defined in the protocol. We can assure that the measurements will by no means delay therapy.

\section{STATISTICS}

Detailed methodology for summaries and statistical analyses of the data collected in this study will be documented in a statistical analysis plan, which will be finalised before database closure and will be under version control by the CTU, University Hospital of Basel.

\section{Hypothesis}

We hypothesise that the infusion of dexmedetomidine compared with propofol over the designated period of time leads to shorter duration and diminished severity of delirium.

The two-sided statistical null hypothesis to be tested for the primary endpoint: as an addition to standard therapy, there is no difference regarding the duration of delirium between patients receiving dexmedetomidine compared with propofol.

\section{Determination of sample size}

Sample size was estimated to be able to show the superiority of dexmedetomidine compared with propofol regarding the duration of delirium in hours.

Sample size calculation was based on pilot data on the number of intensive care shifts (three per day) in which delirium was detected in 118 patients with hyperactive or mixed type delirium on the ICU. Delirium duration was calculated as (number of observed shifts with delirium minus 1$) \times 8$ hours. The first shift with delirium will be subtracted because it is an inclusion criterion, and patients will be randomised to either drug after delirium has been diagnosed for the first time. Multiplication by 8 hours is due to the shift duration. Furthermore, it is assumed that these patients were treated with propofol and that treatment with dexmedetomidine would lead to a reduction of the delirium duration by $\theta \%$ (relative effect).

Sample size was calculated with a semiparametric resampling method as suggested by Davison and Hinkley 


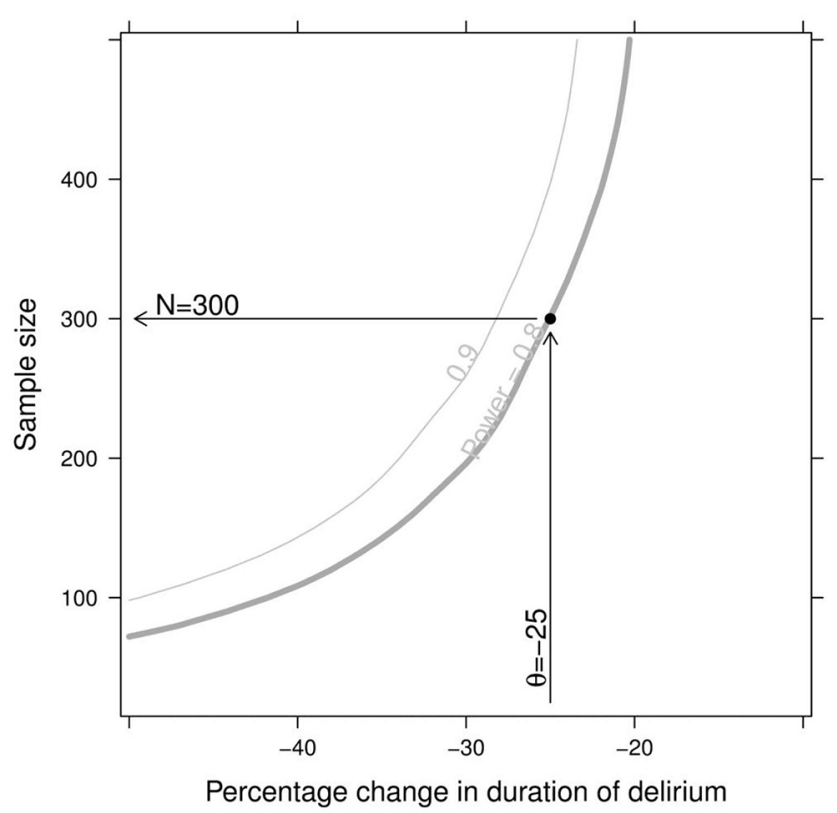

Figure 2 Total sample size (number of patients, not including dropouts) needed to be able to show the superiority of dexmedetomidine to propofol regarding the duration of delirium (hours), depending on the relative effect (\% reduction). The numbers on the curves show the corresponding power. An example is shown for a relative effect of $-25 \%$ for patients with dexmedetomidine compared with patients with propofol, and a power of $80 \%$. The curves are smoothed and for illustration only.

(1997). ${ }^{33}$ This allows to simultaneously account non-parametrically for the distribution of delirium duration in the pilot data set (which is fairly skewed) and parametrically for the treatment shift, $\theta$. Each sample size, $\mathrm{n}_{\mathrm{i}=}$ ${ }_{1-49}=20$, 500, was evaluated by sampling 9999 times $n_{i}$ individual patients with replacement from the pilot data. Half of the patients were randomly assigned to dexmedetomidine and propofol. Thereby, different relative effects, $\theta$ (percentage reduction in delirium duration), ranging from -50 to -10 , were applied to the patients in the dexmedetomidine group by multiplying their delirium durations with a factor $(1+\theta / 100)$. Thereafter, a Wilcoxon rank-sum test was used to test for a difference between the two groups. Sample size was set to ensure at least $80 \%$ power $(1-\beta=0.8)$, at a significance level of $5 \%$ $(\alpha=0.05)$.

For this study, assuming a relative effect of $\theta=-25 \%$ (which corresponds to a reduction in the median duration of delirium by 24 hours), 316 patients should be recruited to ensure 300 evaluable patients considering a dropout rate of $5 \%$. figure 2 presents the sensitivity of sample size with respect to the expected reduction in the duration of delirium.

\section{Planned analyses}

Datasets to be analysed, analysis populations

We will conduct an ITT and and a per protocol analysis.

The ITT set will include all patients randomised to dexmedetomidine or propofol. According to the ITT principle, patients will be analysed according to the randomised treatment.

The PP set will include all patients from the ITT set who meet the inclusion criteria, do not meet any of the exclusion criteria and do not have a major protocol violation (eg, inclusion criteria not met or exclusion criteria met). Patients not receiving the randomised treatment will be analysed according to the received treatment.

\section{Primary analysis}

The primary outcome, duration of the delirium, will be compared between patients treated with dexmedetomidine and patients treated with propofol by a Wilcoxon rank-sum test (ie, Mann-Whitney test) applied to the ITT set.

To assess the sensitivity of the result with regard to the analysis used, we will conduct the following sensitivity analyses:

S1: analysis described above applied to the PP set.

S2: a linear model on log-transformed duration of the delirium (instead of the non-parametric Wilcoxon rank-sum test) will be applied to the ITT set.

S3: as S2 but adjusting the treatment effect by including covariates in the model that might also affect the duration of delirium.

\section{Secondary analyses}

Continuous secondary outcomes will be compared between the two trial arms by Wilcoxon rank-sum tests or linear models depending on whether the normality assumption for the residuals will be violated. The lengths of stay (ICU and hospital) will be compared between the two trial arms as the time to discharge using Cox proportional hazards models. Categorical secondary outcomes will be compared between the two trial arms by generalised linear models. All analyses on secondary endpoints will be applied to the ITT set.

The emergence of different sleep-like patterns and/or the presence and changes of patterns of acute encephalopathy during sedation will be analysed descriptively based on EEG characteristics in patients from the dexmedetomidine trial arm.

\section{Deviation(s) from the original statistical plan}

If for whatever reason substantial deviations of the analysis, as outlined in this section, are needed, the protocol will be amended. All deviations of the analysis from the protocol or from the detailed analysis plan will be listed and justified in a separate section of the final statistical report.

\section{Handling of missing data and dropouts}

Missing values on the primary endpoint will be imputed. A detailed description of the imputation methods and corresponding sensitivity analyses will be specified in the detailed analysis plan. Missing values on secondary endpoint will not be assigned (complete case analyses). 


\section{DATA REGISTRATION}

Data will be entered into a web-based electronic case report form established by the CTU Basel. Paper case report forms will be used in parallel also because of possible technical difficulties.

\section{DATA HANDLING AND MANAGEMENT}

All data from this study will be kept within the Investigator Site File, and only the study team will have access. In case of a patient's ex-post denial of study participation, the data collected will not be used for publication involving neither the corresponding trial nor future trials. In such cases, the data will be destroyed.

All study data will be archived in a designated place on our Surgical ICU at the University Hospital of Basel for a minimum of 10 years after study termination or premature termination of the clinical trial. We plan to store the data also within an electronic case report form (eCRF).

\section{MONITORING}

We have appointed an experienced study nurse of the University Hospital of Basel to be responsible for trial monitoring focusing on data entry.

No regular monitoring visits at the investigator's site prior to the start of the study are planned by the Sponsor. Monitoring will commence with the trial initiation visit, followed by regular monitoring visits within time frames that will have to be determined in the beginning. A daily monitoring of eCRF performance will be conducted by a member of the study team.

The source data/documents are accessible to monitors, and questions are answered during possible monitoring.

\section{ETHICAL JUSTIFICATION}

Due to the nature of delirium, patients eligible for study participation are not able to give their consent. As described above, we will seek the patient's approval for use of collected data for our publication after the delirium has resolved.

Delirium is a serious condition calling for immediate diagnosis and therapy. Because ICU length of stay is associated with patient morbidity and mortality, we chose to investigate a therapeutic approach that might reduce the duration and severity of delirium, thus leading to shorter ICU and hospital stays.

By achieving our goal, we can positively influence patient well-being after severe medical condition and, most importantly, promote a reduction in patient morbidity and mortality and enhance patient satisfaction. This in turn would have a positive impact on our society, and on the economy.

\section{ENROLMENT}

Patients from both Surgical and Medical ICUs of the University Hospital Basel are primarily scheduled for trial participation. The study is planned to begin in January 2017 and will continue for a 3-year period.

\section{TRIAL MANAGEMENT AND ORGANISATION}

The trial will be organised and managed by the research team of the Surgical ICU, University Hospital of Basel, in collaboration with the staff of the Surgical ICU, the Medical ICU and the Department for Clinical Neurophysiology, Epilepsy and Movement Disorders, University Hospital of Basel. The latter will be responsible for carry out the EEG and analysis. Monitoring will be provided by an experienced study nurse who is not member of the research team. The statistical research plan and statistical analysis as well as the establishment of the eCRF will be ensured by the CTU Basel.

Coenrolment of study participants in other clinical trials is basically allowed but will have to be discussed among the competing research teams prior to randomisation.

\section{INSURANCE}

Insurance will be provided by the sponsor through the liability insurance of the University Hospital Basel.

\section{DATA SHARING AND PUBLICATION}

Study results will be communicated to patients based on expected speed-up in convalescence from delirious state. During the ongoing study and until publication, there will be no public access to the data. We plan to publish the data in a major peer-reviewed clinical journal.

A public description of the study in German will be available on the SNCTP after gaining approval for study conduction from the competent ethics committee.

\section{TIMELINE}

Study conductance is planned as follows (table 4).

\begin{tabular}{ll}
\hline Table 4 & \\
\hline Year & Procedure \\
\hline 2016 & Approval from competent ethics committee \\
& Trial registration \\
& Funding application \\
& Purchase of EEG device \\
& Establishment of eCRF \\
& Development of monitoring plan \\
& Medical staff study training \\
& Inclusion of 316 patients \\
2017-2019 & Follow-up of 316 patients \\
& Annual safety report \\
2019 & Data analysis \\
& Writing and submission of manuscript for \\
& publication \\
\hline
\end{tabular}


Table 5 Calculation of average cost of dexmedetomidine study treatment per day

\begin{tabular}{|c|c|c|}
\hline $\begin{array}{l}\text { Price of dexmedetomidine: } \\
200 \mu \mathrm{g} / 2 \mathrm{~mL}, 1 \mathrm{box}=\text { five } 2 \mathrm{~mL} \text { ampoules }\end{array}$ & CHF & 143.24 \\
\hline $\begin{array}{l}\text { Price of dexmedetomidine per } \\
\text { ampoule }\end{array}$ & CHF & 28.65 \\
\hline Patient weight: assumed average & kg & 80.00 \\
\hline $\begin{array}{l}\text { Duration of dexmedetomidine } \\
\text { infusion: } 20: 00-06: 00\end{array}$ & hrs & 10.00 \\
\hline
\end{tabular}

Average dose of dexmedetomidine

per hour: Bolus of $0.7 \mu \mathrm{g} / \mathrm{kg} / \mathrm{hour}$, followed by $0.2-1.4 \mu \mathrm{g} / \mathrm{kg} / \mathrm{hour}$ for desired level of sedation

Bolus of $0.7 \mu \mathrm{g} / \mathrm{kg} / \mathrm{hour}$ (first hour) (median; 9 hours)
- Continuous infusion of $0.8 \mu \mathrm{g} / \mathrm{kg} / \mathrm{hour}$

$\begin{array}{ll}\mu \mathrm{g} & 56.00 \\ +\mu \mathrm{g} & 576.00 \\ =\mu \mathrm{g} & 632.00\end{array}$

\section{FINANCES}

\section{Funding}

Approval of financial support over CHF 92500 was given by the Research Foundation of the University Hospital Basel.

- Study drug/personnel/laboratory will be financed by the above-mentioned grant approved by the Research Foundation of the University Hospital Basel.

- All other drugs used during the study are part of the routine treatment of patients with delirium. No additional costs will arise.

There has not been or will be any influence on trial design and conductance by any funding sources.

\section{Rationale: dexmedetomidine as considerable cost factor}

Calculation of average cost of dexmedetomidine study treatment per day (Table 5; for price overview, see online supplementary appendix).

\section{Calculation of average dexmedetomidine costs:}

To administer the dose of $632 \mu \mathrm{g}$ of dexmedetomidine calculated for 1 day, four ampoules will need to be used: $632 / 200=3.16$ ampoules. Therefore, the total cost per day attributed to dexmedetomidine is $4 * 28.65=C H F$ 114.6.

In the final analysis, we could compare the cost difference of dexmedetomidine to propofol with the average ICU length of stay defined by the average cost of an ICU treatment/24hours in Switzerland.

\section{DISCUSSION}

\section{Trial rationale}

We hypothesise that the infusion of dexmedetomidine compared with propofol over the designated period of time will lead to a shorter duration and diminished severity of delirium. The results of this study may lead to better algorithms for the treatment of delirium, which could improve clinical care for patients, reduce the burden of family members and protect the patient's longterm autonomy and health.

\section{Population}

We include patients admitted to the ICU suffering from hyperactive or mixed delirium that calls for treatment independent from the study.

\section{Intervention}

There is limited but promising evidence that dexmedetomidine shortens the duration of hyperactive delirium, diminishes delirium severity and induces a sleep-like pattern that shifts sleep activity to nightly hours. In our trial, we aim to confirm the superiority of dexmedetomidine over propofol for the delirium treatment. The most common side effects of both study drugs, dexmedetomidine and propofol (comparator), are bradycardia and hypotension. Study data suggest that dexmedetomidine compared with propofol reveals a safer risk profile concerning these side effects.

\section{Comparator}

We chose propofol as active comparator as it has been established as a standard sedative agent for continuous infusion in compromised patients due to limited self-control under the influence of hyperactive and mixed delirium. Benzodiazepines as other possible comparators stand under strong suspicion to induce delirium.

\section{Outcome}

Because of evidence that dexmedetomidine shortens the duration of delirium, we chose to evaluate the duration of delirium in hours to be able to calculate a significant reduction in its duration and thereby prove our hypothesis. The number of delirium free days at day 28 will be used to compensate for competing risks like intensive care mortality.

\section{Sample size}

As described above, sample size was estimated to be able to show the superiority of dexmedetomidine compared with propofol regarding the duration of delirium in hours.

\section{Perspective}

The Basel ProDex study aims to improve quality of delirium treatment by implementation of dexmedetomidine into the treatment regime based on high quality data.

\section{Trial status}

The ethics committee granted approval of this study in September 2016. Inclusion of first patient planned for March 2017.

\section{Study protocol publication}

The manuscript was written by following the Spirit Checklist.

Acknowledgements We thank Allison Dwileski for language contribution.

Contributors The authors have contributed, are contributing and will contribute to this manuscript/study conductance/publication of study results as follows: conception or design of the work: MS, SR, RS, LAS and AH. Data collection: KL, SZ, RS, MS, AB and AH. Data analysis and interpretation: SvF, MS, SR, RS, LAS, SM and 
AH Drafting the article: AH, LG and MS. Critical revision of the article: all declared authors. Final approval of the version to be published: all declared authors.

Competing interests None declared.

Ethics approval Ethics Committee (EKNZ: Ethikkommission Nordwest- und Zentralschweiz)

Provenance and peer review Not commissioned; externally peer reviewed.

Open Access This is an Open Access article distributed in accordance with the Creative Commons Attribution Non Commercial (CC BY-NC 4.0) license, which permits others to distribute, remix, adapt, build upon this work non-commercially, and license their derivative works on different terms, provided the original work is properly cited and the use is non-commercial. See: http://creativecommons.org/ licenses/by-nc/4.0/

(c) Article author(s) (or their employer(s) unless otherwise stated in the text of the article) 2017. All rights reserved. No commercial use is permitted unless otherwise expressly granted.

\section{REFERENCES}

1. Mo Y, Zimmermann AE. Role of dexmedetomidine for the prevention and treatment of delirium in intensive care unit patients. Ann Pharmacother 2013;47.

2. Page VJ, McAuley DF. Sedation/drugs used in intensive care sedation. Curr Opin Anaesthesiol 2015;28:139-44.

3. Wujtewicz M, Maciejewski D, Misiołek $\mathrm{H}$, et al. Use of dexmedetomidine in the adult intensive care unit. Anaesthesiol Intensive Ther 2013;45:235-40.

4. Devlin JW, Al-Qadhee NS, Skrobik Y. Pharmacologic prevention and treatment of delirium in critically ill and non-critically ill hospitalised patients: a review of data from prospective, randomised studies. Best Pract Res Clin Anaesthesiol 2012;26:289-309.

5. Maldonado JR, Wysong A, van der Starre PJ, et al. Dexmedetomidine and the reduction of postoperative delirium after cardiac surgery. Psychosomatics 2009;50:206-17.

6. Barr J, Fraser GL, Puntillo K, et al. Clinical practice guidelines for the management of pain, agitation, and delirium in adult patients in the intensive care unit. Crit Care Med 2013;41:263-306.

7. Reade MC, O'Sullivan K, Bates S, et al. Dexmedetomidine vs. haloperidol in delirious, agitated, intubated patients: a randomised open-label trial. Crit Care 2009;13:R75.

8. Ely EW, Stephens RK, Jackson JC, et al. Current opinions regarding the importance, diagnosis, and management of delirium in the intensive care unit: a survey of 912 healthcare professionals. Crit Care Med 2004;32:106-12.

9. Hollinger A, Siegemund M, Goettel N, et al. Postoperative delirium in cardiac surgery: an unavoidable menace? J Cardiothorac Vasc Anesth 2015;29:1677-87.

10. Friedman JI, Soleimani L, McGonigle DP, et al. Pharmacological treatments of non-substance-withdrawal delirium: a systematic review of prospective trials. Am J Psychiatry 2014;171:151-9.

11. Ji F, Li Z, Nguyen H, et al. Perioperative dexmedetomidine improves outcomes of cardiac surgery. Circulation 2013;127:1576-84.

12. Reade MC, Eastwood GM, Bellomo R, et al. Effect of Dexmedetomidine added to Standard Care on Ventilator-Free Time in Patients with agitated Delirium: a Randomized clinical trial. JAMA 2016;315:1460-8.

13. Teegarden BM, Prough DS. What to do when Haloperidol fails to treat agitated delirium: is Dexmedetomidine the next step? Crit Care Med 2016;44:1426-8.

14. Carrasco G, Baeza N, Cabré L, et al. Dexmedetomidine for the treatment of Hyperactive Delirium Refractory to Haloperidol in Nonintubated ICU Patients: a Nonrandomized Controlled trial. Crit Care Med 2016;44:1295-306.

15. Cruickshank M, Henderson L, MacLennan G, et al. Alpha-2 agonists for sedation of mechanically ventilated adults in intensive care units: a systematic review. Health Technol Assess 2016;20:1-118.

16. Chen K, Lu Z, Xin YC, et al. Alpha-2 agonists for long-term sedation during mechanical ventilation in critically ill patients. Cochrane Database Syst Rev 2015;1:CD010269.

17. Huupponen E, Maksimow A, Lapinlampi $P$, et al. Electroencephalogram spindle activity during dexmedetomidine sedation and physiological sleep. Acta Anaesthesiol Scand 2008;52:289-94.

18. Alexopoulou C, Kondili E, Diamantaki E, et al. Effects of dexmedetomidine on sleep quality in critically ill patients: a pilot study. Anesthesiology 2014;121:801-7.
19. Hollinger A, Siegemund M, Goettel N, et al. Postoperative delirium in cardiac surgery: an unavoidable menace? J Cardiothorac Vasc Anesth 2015;29:1677-87.

20. Tashkandi J. My patient is allergic to eggs, can i use propofol? A case report and review. Saudi J Anaesth 2010;4:207-8.

21. Khan BA, Guzman O, Campbell NL, et al. Comparison and agreement between the Richmond Agitation-Sedation Scale and the Riker Sedation-Agitation Scale in evaluating patients' eligibility for delirium assessment in the ICU. Chest 2012;142:48-54.

22. Fong TG, Tulebaev SR, Inouye SK. Delirium in elderly adults: diagnosis, prevention and treatment. Nat Rev Neurol 2009;5:210-20.

23. Lipowski ZJ. Transient cognitive disorders (delirium, acute confusional states) in the elderly. Am J Psychiatry 1983;140:1426-36.

24. Fong TG, Tulebaev SR, Inouye SK. Delirium in elderly adults: diagnosis, prevention and treatment. Nat Rev Neurol 2009;5:210-20.

25. Asserhøj LL, Mosbech H, Krøigaard M, et al. No evidence for contraindications to the use of propofol in adults allergic to egg, soy or peanut $\dagger$. Br J Anaesth 2016;116:77-82.

26. Balas MC, Vasilevskis EE, Burke WJ, et al. Critical care nurses' role in implementing the "ABCDE bundle" into practice. Crit Care Nurse 2012;32:35-48.

27. Trogrlić Z, van der Jagt M, Bakker J, et al. A systematic review of implementation strategies for assessment, prevention, and management of ICU delirium and their effect on clinical outcomes. Crit Care 2015;19:157.

28. van Eijk MM, van Marum RJ, Klijn IA, et al. Comparison of delirium assessment tools in a mixed intensive care unit. Crit Care Med 2009;37:1881-5.

29. Gusmao-Flores D, Salluh JI, Dal-Pizzol F, et al. The validity and reliability of the portuguese versions of three tools used to diagnose delirium in critically ill patients. Clinics 2011;66:1917-22.

30. Nishimura K, Yokoyama K, Yamauchi N, et al. Sensitivity and specificity of the Confusion Assessment Method for the Intensive Care Unit (CAM-ICU) and the Intensive Care Delirium Screening Checklist (ICDSC) for detecting post-cardiac surgery delirium: a single-center study in Japan. Heart Lung 2016;45:15-20.

31. Bergeron N, Dubois MJ, Dumont M, et al. Intensive Care Delirium Screening Checklist: evaluation of a new screening tool. Intensive Care Med 2001;27:859-64.

32. Brummel NE, Vasilevskis EE, Han JH, et al. Implementing delirium screening in the ICU: secrets to success. Crit Care Med 2013;41:2196-208.

33. Davison AC, Hinkley DV. Bootstrap methods and their application. Camridge, UK: Cambridge University Press, 1997.

34. Carella F, Travaini G, Contri P, et al. Cerebral complications of coronary by-pass surgery. A prospective study. Acta Neurol Scand 1988;77:158-63.

35. Keating GM, Hoy SM, Lyseng-Williamson KA. Dexmedetomidine: a guide to its use for sedation in the US. Clin Drug Investig 2012;32:561-7

36. Pasin L, Landoni G, Nardelli P, et al. Dexmedetomidine reduces the risk of delirium, agitation and confusion in critically III patients: a meta-analysis of randomized controlled trials. J Cardiothorac Vasc Anesth 2014;28:1459-66.

37. Leung JM, Sands LP, Rico M, et al. Pilot clinical trial of gabapentin to decrease postoperative delirium in older patients. Neurology 2006;67:1251-3.

38. Reardon DP, Anger KE, Adams CD, et al. Role of dexmedetomidine in adults in the intensive care unit: an update. Am J Health Syst Pharm 2013;70:767-77.

39. Sockalingam S, Parekh N, Bogoch II, et al. Delirium in the postoperative cardiac patient: a review. J Card Surg 2005;20:560-7.

40. Mu DL, Wang DX, Li LH, et al. High serum cortisol level is associated with increased risk of delirium after coronary artery bypass graft surgery: a prospective cohort study. Crit Care 2010;14:R238.

41. Hakim SM, Othman Al, Naoum DO. Early treatment with risperidone for subsyndromal delirium after on-pump cardiac surgery in the elderly: a randomized trial. Anesthesiology 2012;116:987-97.

42. Tagarakis G, Tsolaki-Tagaraki F, Tsolaki M, et al. The role of SOAT-1 polymorphisms in cognitive decline and delirium after bypass heart surgery. Clin Res Cardiol 2007;96:600-3.

43. Tagarakis GI, Voucharas C, Tsolaki F, et al. Ondasetron versus haloperidol for the treatment of postcardiotomy delirium: a prospective, randomized, double-blinded study. J Cardiothorac Surg 2012;7:25.

44. Mu JL, Lee A, Joynt GM. Pharmacologic agents for the prevention and treatment of delirium in patients undergoing cardiac surgery: systematic review and metaanalysis. Crit Care Med 2015;43:194-204.

45. Shehabi $\mathrm{Y}$, Nakae $\mathrm{H}$, Hammond $\mathrm{N}$, et al. The effect of dexmedetomidine on agitation during weaning of mechanical 
ventilation in critically ill patients. Anaesth Intensive Care 2010;38:82-90.

46. Sarasin DS, Ghoneim MM, Block RI. Effects of sedation with midazolam or propofol on cognition and psychomotor functions. $J$ Oral Maxillofac Surg 1996;54:1187-93.

47. Riker RR, Shehabi Y, Bokesch PM, et al. Dexmedetomidine vs midazolam for sedation of critically ill patients: a randomized trial. JAMA 2009;301:489-99.

48. Sheldon T. Study of rivastigmine for delirium in intensive care is stopped after deaths. BMJ 2010;340:c2895.

49. Al-Aama T, Brymer C, Gutmanis I, et al. Melatonin decreases delirium in elderly patients: a randomized, placebo-controlled trial. Int $J$ Geriatr Psychiatry 2011;26:687-94.

50. Reade MC, Finfer S. Sedation and delirium in intensive care. $N$ Engl $J$ Med 2014;370:1567.
51. Hudetz JA, Patterson KM, lqbal Z, et al. Ketamine attenuates delirium after cardiac surgery with cardiopulmonary bypass. $J$ Cardiothorac Vasc Anesth 2009;23:651-7.

52. Shehabi $\mathrm{Y}$, Grant $\mathrm{P}$, Wolfenden $\mathrm{H}$, et al. Prevalence of delirium with dexmedetomidine compared with morphine based therapy after cardiac surgery: a randomized controlled trial (DEXmedetomidine COmpared to Morphine-DEXCOM Study). Anesthesiology 2009;111:1075-84.

53. Park JB, Bang SH, Chee HK, et al. Efficacy and safety of dexmedetomidine for postoperative delirium in adult cardiac surgery on cardiopulmonary bypass. Korean J Thorac Cardiovasc Surg 2014;47:249-54

54. Devlin JW, Marquis F, Riker RR, et al. Combined didactic and scenario-based education improves the ability of intensive care unit staff to recognize delirium at the bedside. Crit Care 2008;12:R19. 